\title{
COMPARISON OF MIRROR THERAPY AND MOTOR RELEARNING PROGRAM IN IMPROVING THE UPPER LIMB MOTOR FUNCTION OF PATIENTS WITH STROKE
}

\author{
Rabia Rauf, Abdul Rashad*, Alisha Noreen**, Rabia Intikhab***, Tehreem Anis Suleman***, Seemab Mughal**** \\ Royal College of Physiotherapy, Rahim Yar Khan, Pakistan, *United College of Physical Therapy, Karachi Pakistan, **Iqra University, Karachi Pakistan,

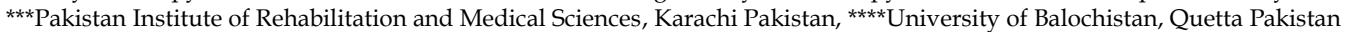

\begin{abstract}
Objective: To compare the effectiveness of mirror therapy and motor relearning program for improving the upper limb motor function in stroke patients.

Study Design: A randomized control trial with registration number IRCT20200316046791N1 (IRCT).

Place and Duration of Study: Al-Noor Hospital and Al Jannat Medicare, Rahim Yar Khan Pakistan, from Jan to Jul 2020.

Methodology: Sample size consisted of 30 patients recruited by convenient sampling. Patients were randomized into two groups. Both groups received 5 sessions a week for 6 weeks. Group A received Motor Relearning Program while the treatment of group B was Mirror Therapy. Motor part of Fugl Meyer Assessment was used as an outcome measure. Non parametric Friedman test was used for within group analysis, while for between group analyses Karuskal-Wallis test was used.

Results: The total participants who suffer from left hemiplegia were 22 whereas the patients having right hemiplegia were 8. The mean age of participants in both groups was $53.80 \pm 7.6$ group A median (interquartile range) at pre-assessment was 5.00 (3) at mid-assessment median=15.00 (4) while at post assessment median was 25.00 (6). Group B median at baseline was 6.00 (4), at mid-assessment median=14.00 (9), post assessment median was 17.00 (13). Mid and post assessment comparison showed both treatments improved the motor function. Statistically the results were non-significant $(p>0.005)$. Both treatments improved the motor function in stroke patients.

Conclusion: There is no significant difference between the effectiveness of Mirror Therapy and Motor relearning program in improving the upper limb motor function of stroke patients.
\end{abstract}

Keywords: Motor function, Stroke, Upper extremity.

This is an Open Access article distributed under the terms of the Creative Commons Attribution License ( https://creativecommons.org/licenses/by-nc/4.0/), which permits unrestricted use, distribution, and reproduction in any medium, provided the original work is properly cited.

\section{INTRODUCTION}

Stroke is a clinical syndrome which is defined as quickly developed signs of focal or global disruption of functions of cerebrum, lasting for greater than 24 hours. It may lead to death, with none obvious causes other than vascular origin ${ }^{1}$. Most common signs and symptoms associated with stroke are weakness on one side of body and speech problems ${ }^{2}$.

Globally stroke is considered as second most leading cause of death and disability ${ }^{3}$. According to the global burden of disease in 2013 men have continuously greater frequency of ischemic stroke than women. In Pakistan the true incidence of stroke is not reported as there is no specific population based study conducted yet. The anticipated stroke cases in Pakistan are 250/100,0005 Arm and hands help in various activities of daily living including manipulation of objects, special type of grasping and control of proximal and distal joints to perform different goals. The patients who experienced upper limb impairment due to stroke lost their ability to perform these specific tasks of grasping

Correspondence: Dr Rabia Rauf, Royal College of Physiotherapy, Rahim Yar Khan, Pakistan

Received: 02 Feb 2021; revised received: 21 Apr 2021; accepted: 30 Apr 2021 and manipulation. To treat the stroke related movement impairments it is important to identify underlying causes and further proper investigation of the effective treatment option ${ }^{6}$. Numerous patients (41-45\%) endure chronic motor disabilities and restrictions in activities of everydayliving indeed after broad neurological recovery. There are different researches which have used different interventions with variations in treatment duration and protocols to evaluate the best effective treatment for stroke. For the stroke Rehabilitation concern the main therapeutic methods for patients are Brunnstrom, Bobath therapy proprioceptive neuromuscular facilitation (PNF), motor relearning program (MRP), Mirror therapy as well as constrained induced movement therapy $(\mathrm{CIMT})^{7}$. In the beginning the purpose of Mirror Therapy was to reduce the pain of phantom limb in persons with amputations. The mirror reflection of sound limb gave the patients a sensation of moving both arms which results in reduction of pain sensation. Ramachandran explained the mechanism of relearning process in the brain which is being unlearned $^{8}$. The motor relearning program (MRP) was devised by Janet Carr and Roberta Shepherd who was the Australian physiotherapists by profession. To improve motor control it is a type of task-oriented approach, 
which focuses on the relearning of daily activities. MRP powerfully based on concepts in kinesiology that stresses on distributed motor control model. Motor relearning program consists of four steps including analysis of the task, Practice of missing component, and practice of task and last one is about Transference of training 9 . The Fugl Meyer is a properly designed, convenient and productive clinical examination tool that has been tried broadly within the stroke populace. It has excellent intra-rater, inter-rater and construct validity ${ }^{10}$. The study was aimed to compare the effectiveness of motor relearning program and mirror therapy in stroke patients. There is a need to incorporate easy, simple, economic, patient directed and effective treatment techniques to enhance recovery following stroke. There is less data available on comparison between motor relearning and mirror therapy in the upper limb in the past. Researches on either the effects of MRP or the effects of mirror therapy can be found. That is why this particular kind of research will contribute in stroke rehabilitation.

\section{METHODOLOGY}

This single blind (assessor) randomized trial was conducted from January to July 2020 after ethics approval from Isra University ASRC-055/2020). The data was collected from Al Noor Hospital and Al Jannat Medicare Rahim Yar Khan. Sample was calculated by an online calculator OpenEpi tool and consisted of 30 patients; previous study's prevalence was used for sample calculation ${ }^{11}$. Sampling technique was non-probability convenient sampling After informed consent Patients withischemic and hemorrhagic stroke, in age range of 40-65 years, having unaffected extremity were included in the study. Patients who were not mentallystable, having any musculoskeletal impairment and patients with Mini Mental State Examination <23 were excluded. All assessments before and after the intervention were performed by independent assessor and only eligible subjects were enrolled. Randomization was computerized with concealed allocation sequence carried out at an external sitethrough blind acessor. Treatment protocol included 5 sessions a week for 6 weeks and the session duration was 40 minutes. Group A of this study received MRP (motor relearning program) treatment which included different types of task specific exercises, reaching and manipulation. Practice of wrist flexion and extension with holding different objects, pronation/supination, and opposition of thu$\mathrm{mb}$ for paretic upper limb. Activities were started with simple tasks and less repetitions and were progressed to more complex tasks andrepetitions. The group B was treated with mirror therapy. The activities performed in this group were in front of mirror and with the non-paretic limb. The mirror size was $20 \times 25$. Visual or verbal instructions were given about the movement. The mirror was placedin the midline ofpatient ${ }^{12}$. The unaffected limb was positioned similar to that of the affected limb. Next, patients were instructed to observe the mirror reflection for one to two minutes, trying to visualize the mirror image as the affected limb. Unilateral motor exercises with the non-affected limb were performed. All the patients were assessed before treatment (pre assessment), at $3^{\text {rd }}$ week (mid assessment) and after $6^{\text {th }}$ week (post assessment) with the outcome measure Modified Fugl Meyer Asse-ssment scale.

The data analysis was made through SPSS-20. Data was not normally distributed so non-parametric tests were used. For pre-mid and post intra group comparison, friedman test was used. While for inter group comparison of both groups was made using KaruskalWallis test.

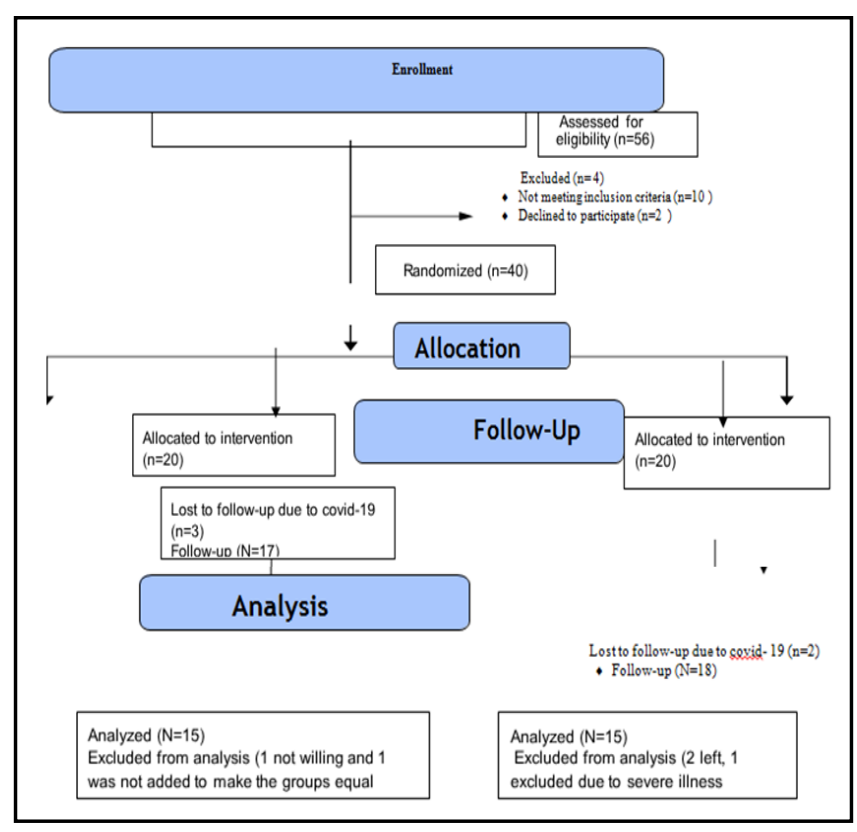

Figure-1: The consort diagram for RCT.

\section{RESULTS}

Out of the total 30 participants male and femalein both groups were $21(70 \%)$ and $9(30 \%)$ respectively. The total participants who suffer from left hemiplegia were $22(73.33 \%)$ where as the number of patients having right hemiplegia was $8(26.66 \%)$. The mean age of participants in both groups was $53.80 \pm 7.6$ (table-I). 
The results showed statistically significant improvement in motor function of stroke patients from baseline to post assessment in both groups $(p<0.005)$. For group A Fugl Meyer median (IQR) at pre-assessment was 5.00 (3), at mid-assessment median 15.00 (4) while at post assessment median was 25.00 (6) whereas for group B median at pre-assessment was 6.00 (4), midassessment median 14.00 (9) at post assessment median was 17.00 (13) (table-II). There was no significant difference presentat baseline between two groups. Preassessment and post assessment median comparison showed both treatments improved the motor function in stroke patients and there was no statistically significant difference present in terms of Fugl Meyer scores $(p>$ 0.005) (table-III).

Table-I: Gender and affected side distribution of study participants.

\begin{tabular}{l|c|c}
\hline Demographics & $\begin{array}{c}\text { Group-A with } \\
\text { Percentage }\end{array}$ & $\begin{array}{c}\text { Group-B with } \\
\text { Percentage }\end{array}$ \\
\hline Male & $11(73.3 \%)$ & $10(66.7 \%)$ \\
\hline Female & $4(26.7)$ & $5(33.3)$ \\
\hline Left Hemiplegia & $10(66.7 \%)$ & $12(80 \%)$ \\
\hline Right Hemiplegia & $5(33.3 \%)$ & $3(20 \%)$ \\
\hline
\end{tabular}

Table-II: Within group comparison of fugl meyer score using non-parametric friedman test.

\begin{tabular}{l|c|c|c|c}
\hline & PRE & MID & POST & \multirow{2}{p}{$\begin{array}{c}\boldsymbol{p} \text { - } \\
\text { value }\end{array}$} \\
\cline { 2 - 4 } & $\begin{array}{c}\text { Median } \\
\text { (IQR) }\end{array}$ & $\begin{array}{c}\text { Median } \\
\text { (IQR) }\end{array}$ & $\begin{array}{c}\text { Median } \\
\text { (IQR) }\end{array}$ & \\
\hline Group A & $5(3)$ & $15(4)$ & $25(6)$ & $<0.001$ \\
\hline Group B & $5(4)$ & $14(9)$ & $17(13)$ & $<0.001$ \\
\hline
\end{tabular}

Table-III: Post hoc analysis of both groups by wilcoxon test.

\begin{tabular}{l|c|c|c}
\hline & $\begin{array}{c}\text { PRE vs. } \\
\text { MID }\end{array}$ & $\begin{array}{c}\text { MID vs. } \\
\text { POST }\end{array}$ & $\begin{array}{c}\text { POST vs. } \\
\text { PRE }\end{array}$ \\
\hline Group A & 0.001 & 0.001 & 0.001 \\
\hline Group B & 0.001 & 0.001 & 0.001 \\
\hline
\end{tabular}

Table-IV: Between group comparison of fugl meyer score using karuskal-wallis test.

\begin{tabular}{l|c|c|c}
\hline & Group A & Group B & $\begin{array}{c}p- \\
\text { value }\end{array}$ \\
\cline { 2 - 3 } & Median (IQR) & Median (IQR) & 0.33 \\
\hline PRE & $5(3)$ & $6(4)$ & 0.41 \\
\hline MID & $15(4)$ & $14(9)$ & 0.12 \\
\hline POST & $25(6)$ & $17(13)$ & \multirow{2}{*}{} \\
\hline
\end{tabular}

\section{DISCUSSION}

The purpose of this RCT was to compare the effects of two different therapeutic interventions which can be used to enhance themotor function after stroke. Motor relearning program and Mirror therapy were compared for 6 weeks in 30 stroke patients. The mean age of subjects in this study was $53.80 \pm 7.66$. Stroke is a major source of severe long-term disability. More than half of the stroke survivor's age ranged from 65 or above suffer from reduction in mobility ${ }^{13}$.

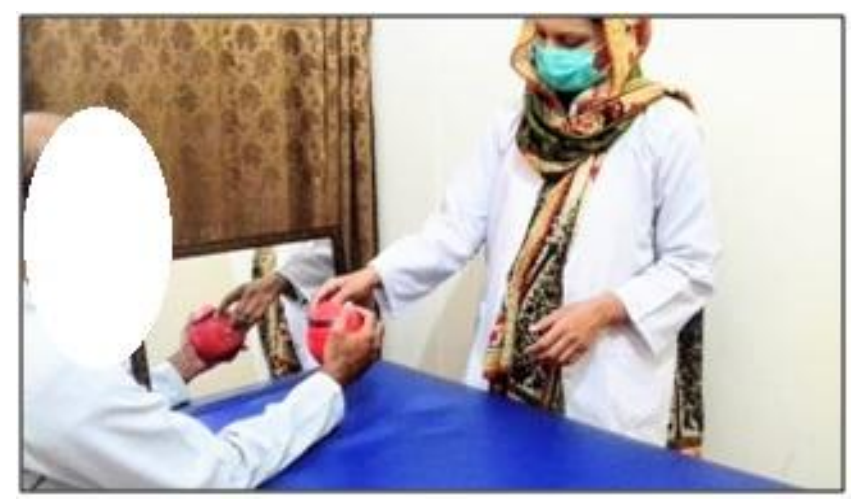

Figure-2: Example of use of mirror for upper limb exercises.

The results of current study were non-significant $(p>0.05)$ comparing the both techniques. There was no significant difference present between two interventions in terms of improving the upper limb motor function of stroke patients. Both interventions improved the upper limb function from baseline to $6^{\text {th }}$ week as median improved in all three assessments. The results of present study are supported by another study which was conducted in India in 2015 The results of that study were in line with current studystated both MRP and Mirror Therapy are effective in improving the motor function afterstroke ${ }^{9}$. One of such study supported the results of current study which compared the effectiveness of task specific exercises and MT, further they evaluated the combine effectiveness of both task specific exercises and MT. The treatments protocol included 30 mints of exercise for 5 days a week for 4 weeks. The significant improvement was seen in all 3 groups. While $3^{\text {rd }}$ group who received combined treatment showed more improvement ${ }^{14}$. Another study related to present study was conducted in Peshawar which compared the effects of MT and MRP for improvement of upper limb motor function in stroke patients. A total of 66 subjects were included in the study and motor assessment scale was used as an outcome measure. This study did not support the present study, their study stated that both Motor relearning program and Mirror therapy are effective in improving the upper limb motor function but MRP is slightly more effective ${ }^{10}$. The differences in results might be due to difference in treatment protocol and geographical changes. The results of our study showed that Motor Relearning program iseffective in improving the upper limb motor function of stroke patients; it is supported by a study conducted in 2017. According to that study 
early administration of motor relearning program training improves the motor function of patients with stroke $^{15}$.

Further the results of present study states that Mirror therapy found to be more effective in enhancing the Upper limb motor function. A similar study supported the current study, which followed MT treatment protocol for 6 weeks, 5 times a week. They included sub-acute stroke patients; the study results showed that early administration of mirror therapy after stroke supports the motor function recovery ${ }^{16}$. The results of our study are supported by another study conducted in Malaysiain 2020. That study stated that Mirror therapy along with conventional treatment is effective to restore the upper limb motor function ${ }^{17}$. This study is supported by a study conducted in Hong Kong, according to that study mirror therapy is more effective than bilateral arm training in improving the motor function of stroke patients ${ }^{18}$.

Both motor relearning program and mirror therapy are effective in the treatment of upper limb motor function of stroke patients. There is no significant difference present in the effectiveness of these techniques.

\section{LIMITATION OF STUDY}

The study was limited to only motor assessment. The data was collected form two settings.

\section{CONCLUSION}

Both interventions are effective in stroke rehabilitation and thereis no significant difference present between the effectiveness of mirror therapy and motor relearning program in improving the upper limb motor function of patients with stroke.

\section{CONFLICT OF INTEREST}

This study has no conflict of interest to be declared by any author.

\section{REFERENCES}

1. Coupland AP, Thapar A, Qureshi MI, Jenkins H, Davies AH. The definition of stroke. J R Soc Med 2017; 110(1): 9-12.

2. Yew KS, Cheng EM. Diagnosis of acute stroke. Ann Fam Med 2015; 91(8): 528-36.

3. Katan M, Luft A. Global burden of stroke. Semin Clin Neuropsy
2018; 38(2): 208-11.

4. Feigin Vl, Norrving B, Mensah GA. Global burden of stroke. Circ Res 2017; 120(3): 439-48.

5. Donnan GA, Fisher M, Macleod M, Davis SM. Stroke. Lancet 2008; 371(9624): 1612-23.

6. Schwarz A, Bhagubai M, Wolterink G, Held JP, Luft AR, Veltink $\mathrm{PH}$. Assessment of upper limb movement impairments after stroke using wearable inertial sensing. J Sens 2020; 20(17): 4770-74.

7. Rehani P, Kumari R, Midha D. Effectiveness of motor relearning programme and mirror therapy on hand functions in patients with stroke - a randomized clinical trial. Int J Ther Rehab Res 2015; 4(3): 20-24.

8. Jan S, Arsh A, Darain H, Gul S. A randomized control trial comparing the effects of motor relearning programme and mirror therapy for improving upper limb motor functions in stroke patients. J Pak Med Assoc 2019; 69(9): 1242-45.

9. Michielsen ME, Selles RW, van der Geest JN, Eckhardt M, Yavuzer G, Stam HJ, et al. Motor recovery and cortical reorganization after mirror therapy in chronic stroke patients: a phase II randomized controlled trial. Neurorehabil Neural Repair 2011; 25(3): 223-33.

10. Singer B, Garcia-Vega J. The Fugl-Meyer upper extremity scale. J Physioth 2017; 63(1): 53-55.

11. Jehan Z, Ali I, Rahman MU, Darain H, Latif A, et al. Prevalence of stroke in hayatabad Peshawar. Ann Allied Health Sci 2018; 4(1): 15-8.

12. Rothgangel A, Braun S, de Witte L, Beurskens A, Smeets R. Mirror therapy. Practical protocol for stroke rehabilitation. 2013 [Internet] Available at: https://www.researchgate.net/ publication/253235147_Mirror_Therapy_Practical_Protocol_for_ Stroke_Rehabilitation

13. Virani , A Alonso, EJ Benjamin, MS Bittencourt, CW Callaway, AP Carson, et al. Heart disease and stroke statistics-2020 update: a report from the American Heart Association. Circulation. 2020 Mar 3; 141(9): e139-596.

14. Khandare SS, Singaravelan RM, Khatri SM. Comparison of task specific exercises and mirror therapy to improve upper limb function in sub-acute stroke patients. J Med Dent Sci 2013; 7(2): 5-14.

15. Min GU, Si-Wei LI, Bao-Jin LI, Cheng LI, Yun QU. Effect of motor relearning programme on motor function recovery of acute stroke patients with hemiplegia. Zhongguo Xian Dai Shen Jing Ji Bing ZaZhi 2017; 17(3): 197.

16. Invernizzi M, Negrini S, Carda S, Lanzotti L, Cisari C, Baricich A. The value of adding mirror therapy for upper limb motor recovery of sub-acute stroke patients: a randomized controlled trial. Eur J Phys Rehabil Med 2013; 49(3): 311-17.

17. Chinnavan E, Ragupathy R, Wah YC. Effectiveness of mirror therapy on upper limb motor functions among hemiplegic patients. Bangladesh J Med Sci 2020; 19(2): 208-13.

18. Fong KN, Ting KH, Chan CC, Li LS. Mirror therapy with bilateral arm training for hemiplegic upper extremity motor functions in patients with chronic stroke. Hong Kong Med J 2019; 25 (Suppl-3): 30-4. 